\title{
SOBRECARGA DE CUIDADORES FAMILIARES DE IDOSOS
}

\section{HOSPITALIZADOS}

Marcos Soares de Arruda; Universidade Federal de São Carlos; marcossarruda@gmail.com

Marcela Naiara Graciani Fumagale Macedo; Universidade Federal de São Carlos; marcelagracianivest@hotmail.com

Sonia Gonçalves da Mota; Universidade Federal de São Carlos; soniagmota_47@hotmail.com

Élen dos Santos Alves; Universidade Federal de São Carlos; elendutra23@gmail.com

Tábatta Renata Pereira de Brito; Universidade Federal de Alfenas; tabatta_renata@hotmail.com Ariene Angelini dos Santos-Orlandi; Universidade Federal de São Carlos; arieneangelini@yahoo.com.br

\section{RESUM0}

Introdução: Doenças crônicas não transmissíveis são comuns em idosos, os quais podem apresentar descompensações e serem hospitalizados. No Brasil, o cuidado ao idoso é culturalmente realizado por um membro familiar. Muitas vezes, esse familiar assume a responsabilidade pelo cuidado de maneira repentina, sem nenhum preparo prévio ou apoio, o que pode resultar em intensa sobrecarga. Nesse contexto, pesquisadores apontam que o esgotamento físico e mental pode influenciar negativamente a qualidade de vida do cuidador. Objetivo: Avaliar a sobrecarga de cuidadores familiares de idosos inseridos no contexto hospitalar. Método: estudo transversal, quantitativo, realizado com 98 cuidadores familiares de idosos que estavam internados na Santa Casa do município de São Carlos-SP. Foram utilizados os seguintes instrumentos: Questionário de caracterização do cuidador, do contexto de cuidado e Inventário de Sobrecarga de Zarit. Na análise descritiva dos dados foram estimadas distribuições de frequências, médias, desvios padrão e proporções. Resultados: a maioria dos cuidadores era do sexo feminino (88,8\%), com média de idade de $54,1 \pm 13,5$ anos e casadas $(74,5 \%)$. Cuidavam do seu progenitor $(45,4 \%)$ ou cônjuge $(32,0 \%)$, há $51,6 \pm 84,3$ meses, durante $16,2 \pm 7,7$ horas por dia, sem treinamento prévio $(81,6 \%)$. Em relação à sobrecarga, o escore médio obtido foi de $34,3 \pm 16,9$ pontos. Todos os cuidadores entrevistados apresentaram algum nível de sobrecarga, sendo a sobrecarga moderada mais prevalente (49,5\%), seguida de moderada a severa $(25,8 \%)$. Conclusão: cuidadores familiares de idosos hospitalizados apresentaram alta prevalência de sobrecarga. Torna-se necessário identificar precocemente essa condição para que sejam planejadas intervenções para melhorar a qualidade de vida desses indivíduos.

Palavras-chave: Cuidadores; Idoso; Esgotamento Psicológico. 